\title{
BMJ Open Doctor, how can we help you? Qualitative interview study to identify key interventions to target burnout in hospital doctors
}

\author{
Gillian Walsh, ${ }^{1,2}$ Blánaid Hayes, ${ }^{2,3}$ Yseult Freeney, ${ }^{4}$ Siobhain McArdle ${ }^{1}$
}

To cite: Walsh G, Hayes B, Freeney $\mathrm{Y}$, et al. Doctor, how can we help you? Qualitative interview study to identify key interventions to target burnout in hospital doctors. BMJ Open 2019:9:e030209. doi:10.1136/ bmjopen-2019-030209

- Prepublication history for this paper is available online. To view these files, please visit the journal online (http://dx.doi. org/10.1136/bmjopen-2019030209).

Received 04 March 2019 Revised 01 August 2019 Accepted 08 August 2019
Check for updates

(C) Author(s) (or their employer(s)) 2019. Re-use permitted under CC BY-NC. No commercial re-use. See rights and permissions. Published by BMJ.

${ }^{1}$ School of Health and Human Performance, Dublin City University, Dublin, Ireland ${ }^{2}$ Research, Royal College of Physicians of Ireland, Dublin, Ireland

${ }^{3}$ Occupational Health, Beaumont Hospital, Dublin, Ireland

${ }^{4}$ Business School, Dublin City University, Dublin, Ireland

Correspondence to

Gillian Walsh;

gillian.walsh4@mail.dcu.ie

\section{ABSTRACT}

Objective To identify priority interventions for the prevention and reduction of work stress and burnout in hospital doctors through analysis of (1) doctors' experiences of work stress and burnout and (2) their preferences with respect to interventions.

Design Qualitative design using semistructured interviews analysed with deductive thematic analysis. Setting Hospitals in Ireland.

Participants 32 hospital doctors (16 practising consultants and 16 doctors in training) from a range of specialties, career stages, hospital types and locations. Results Practical, system-focused interventions were found to be most needed. Challenges with basic entitlements, that is, accessing statutory leave, knowing in advance when leave can be taken and being adequately covered when on leave were identified as requiring urgent attention. Other priority interventions identified were the integration of psychological support in the everyday working environment, time and training for clinical line managers to perform key management activities such as debriefing and education interventions which highlight work stress risks and care pathways, teach self-care and train doctors in how to support one another.

Conclusions Hospital doctors are feeling the effects of greater demand and fewer resources. What they most urgently need is adequate staffing levels, access to statutory leave and adequate cover when on leave. Doctors do not receive the support they need from their clinical line managers, who lack the skills and time to excel as people managers. Organisations should focus on developing clinical management skills across the system. The culture of medicine needs to change from stigmatisation and competitiveness to compassion and collaboration. Organisations, medical schools and professional bodies can steer this change through education.

\section{INTRODUCTION}

Over half of practising physicians report at least one symptom of burnout and this number is growing. ${ }^{2}$ As well as diminished quality of care, ${ }^{34}$ prolonged exposure to burnout can lead to depression and in some cases have devastating consequences for physicians. ${ }^{5}$ A doctor is now twice as likely to take his or her own life as a member of the general
Strengths and limitations of this study

- The main strength of this study is that it puts the physician's voice at the centre of the process of selecting and developing interventions to reduce physician work stress and burnout.

- The study is strengthened by the purposive sample of interviewees selected to capture experiences and preferences of individuals from a range of gender, specialty and career stage, hospital type and geographical location.

- A limitation is that the original pool of volunteers, from which the interviewees were purposively selected, may have been a self-selected group who put themselves forward for interview because of personal experiences.

- A further limitation is that this study did not include non-consultant hospital doctors outside of training programmes and doctors in general practice.

population. ${ }^{6}$ In Ireland, worsening working conditions, resulting from a preceding decade of economic austerity, ${ }^{7}$ and a growing and ageing patient population ${ }^{8}$ have been associated with work stress and burnout, and are likely factors in the current high levels of doctor migration and challenges in physician recruitment. $^{910}$

Work stress occurs when the demands of work exceed an individual's perceived ability to cope in Lazarus and Folkman's transactional model of stress. ${ }^{11}$ In this model, stress is a product of the interaction between the individual and the environment, and this relationship is mediated by cognitive appraisal and coping, whereby coping can be emotion-focused or problem-focused. ${ }^{11}$ Burnout, as described in Maslach et al's model, can be understood as a negative psychological outcome of prolonged work stress with emotional exhaustion and depersonalisation from patients as core features. ${ }^{12}$ Interventions targeted at decreasing work stress and burnout can be classified as primary, secondary or 
Table 1 Taxonomy of interventions for workplace stress modified, with permission, from the original table authored by Clarke and Cooper $^{13}$

\section{Intervention level}

Definition and Description

\section{Primary}

Preventive and proactive

Goal: reducing potential risk or altering the nature of working conditions

the work stressor before workers experience stress-

related symptoms or disease

\section{Secondary}

Ameliorative

Goal: to help equip workers with knowledge, skills and resources to cope with stressful conditions

\section{Tertiary}

Reactive

Goal: to treat, support, compensate and rehabilitate job

\section{Intervention targets Examples}

Stressors at their source, Job redesign, workload reduction, reduction organisation of work and of workflow inefficiencies, improved communications

Employee responses to stressors (perceived stress or strain)

Short-term and enduring adverse health effects of workers with enduring stress-related symptoms and disease
Cognitive-behavioural therapy, stress management training

Return to work programmes, employee assistance programmes, occupational therapy and medical stress interventions tertiary, according to their aim ${ }^{13}$ (see table 1). Primary interventions aim to reduce risk factors for employees. They are usually organisational interventions that tackle work environment factors perceived to contribute to work stress, ${ }^{14}$ for example, changes to work practices, work scheduling and team building. Secondary interventions aim to enhance the ability of workers to manage stressful conditions by increasing awareness, knowledge, skills and coping resources ${ }^{15}$ for example, training in mindfulness and in dealing with difficult patients. Tertiary interventions aim to support recovery and to minimise negative consequences in those already suffering from work stress or burnout, ${ }^{13}$ for example, counselling, rehabilitation and return to work programmes. Tertiary-level interventions are most common and primary-level interventions are the least common ${ }^{16}$ despite governmental policy recommendations that employers adopt a preventive rather than a reactive approach to the management of employee health risks. ${ }^{17} 18$ Combining interventions at primary, secondary and tertiary levels is considered to have high potential value. ${ }^{19}$

While research into prevalence, causes and consequences of burnout in doctors has dominated the literature over the past two decades, more recent calls to shift focus to intervention ${ }^{20}$ have yielded a number of systematic reviews of interventions to prevent and reduce work stress and burnout in the past 3 years. ${ }^{21-24}$ Common findings include poor design of evaluation studies and a concentration on secondary-level, individual-focused interventions. Burnout levels are the most common outcome measures used in the meta-analyses, both for prevention and reduction of burnout. While most reviews found small but significant improvements in burnout levels overall, there remains a lack of clarity around which types of interventions are the most effective. ${ }^{21-23}$ Further and better-quality intervention studies will guide selection of interventions based on efficacy. Listening to doctors themselves, their experiences of work stress and burnout, and their proposed solutions is another valuable exercise that can inform selection of interventions and complement the growing quantitative evidence base.

The physician's voice has been the missing piece in this enquiry, and without it, key factors in development and selection of interventions may be overlooked. It is well established that qualitative data have the potential to shed light on aspects relating to interventions and how they will be received, which quantitative data cannot always uncover. $^{25}$ Several qualitative studies have been conducted as part of intervention evaluations, ${ }^{26} 27$ and others have investigated working conditions, stressors and coping strategies of hospital doctors. ${ }^{28-30}$ However, this is the first qualitative study that aimed to identify priority interventions for work stress and burnout by examining doctors' direct experiences of work stress and burnout and their preferences with respect to such interventions.

\section{METHODOLOGY \\ Design}

A qualitative, semistructured interview design with deductive thematic analysis was used. ${ }^{31}$

\section{Participants}

In a related national survey on work stress and burnout ${ }^{32}$ of hospital doctors, an invitation was extended to participate in further qualitative research. Of those who volunteered, a purposive sample was selected, and interviews were scheduled with 16 practising consultants and 16 doctors in training. After accepting the invitation to participate, one of the scheduled interviews was cancelled by the participant due to work commitments, and an additional participant from that specialty was included. Data saturation was expected to be achieved with less interviews, but 16 was chosen to ensure a representative mix of gender, specialty and career stage (see table 2). An effort was also made to capture experiences of doctors in 


\begin{tabular}{ll}
\hline Table 2 Characteristics of participants \\
\hline Characteristic & Interviewees \\
\hline Gender & 16 female, 16 male \\
Career stage & 16 consultants, 16 doctors in training* \\
Specialty & 6 anaesthetics $†$ \\
& 4 psychiatry \\
& 3 paediatrics \\
& 3 surgery \\
& 3 pathology \\
& 2 emergency \\
& 2 general internal medicine \\
& 2 geriatrics \\
& 1 ophthalmology \\
& 1 urology \\
& 1 obstetrics and gynaecology \\
& 1 respiratory \\
& 1 dermatology \\
& 1 infectious disease \\
& 1 radiology \\
\hline
\end{tabular}

*Doctors in training included basic specialist trainees (equivalent to residency in North America) and higher specialist trainees (equivalent to fellowship in North America).

†From the pool of volunteers that accepted the invitation to participate, to keep the right mix of gender and career stage (which were considered most important) and a good mix of the other criteria, we ended up with a slightly higher number of anaesthetists than other large specialties.

different hospital types and different geographical locations. Written, informed consent was obtained prior to interviewing, and participants were advised of their right to opt out at any stage.

\section{Data collection}

Face-to-face interviews were chosen due to the potentially sensitive nature of the topic. In the interviews, it was necessary to encourage participants to talk openly about their experiences of stress and burnout and coping. Lazarus and Folkman's model of stress ${ }^{11}$ and Maslach $e t$ als model of burnout ${ }^{12}$ were used to inform the design of the interview questions to draw this out. A semistructured interview format was chosen to ensure consistency, and the guide was finalised following three pilot interviews and review of audio files by the full research team. Two main areas were explored: (1) experiences of work stress and burnout and (2) suggested interventions for the prevention and reduction of work stress and burnout. Prior to interviewing, the full research team met to identify and discuss potential biases. Every effort was made to minimise the impact of potential biases throughout the study by keeping note of and reflecting on them throughout the interview, analysis and write-up phases. One-off interviews were conducted by GW at a location chosen by the participants from the following options: their place of work, their home or a private meeting room at the Royal College of Physicians of Ireland (RCPI). There was no one else present. GW is an experienced female researcher with training in qualitative methods and interviewing and was research manager at the RCPI at the time of the study. There was no prior relationship between the interviewer and the interviewees. The interviewer gave a brief personal introduction stating professional background only at the beginning of each interview. Interviews lasted 40-90 min and were audio recorded, transcribed and anonymised prior to analysis. Field notes were taken during interviews. Transcripts were not returned to interviewees for comment unless explicitly requested. Two were requested, provided and returned to researchers with minor amendments.

\section{Analysis}

Thematic analysis, as described by Braun and Clarke, ${ }^{33}$ was chosen for this study. It identifies, analyses and reports patterns or themes across a dataset containing multiple cases (participant interviews). This methodology can be inductive or deductive. We used deductive analysis to identify interventions to tackle work stress and burnout and to prioritise these interventions. The deductive framework used for the analysis is as follows: first, interventions were identified by analysing interviewees' experience of work stress and burnout, identifying key points along the journey from wellness to burnout, which represent opportunities for intervention and transforming these opportunities into directly related, proposed interventions. Second, interventions were identified by capturing those proposed explicitly by interviewees. See figure 1 for an example of analysis by both methods. Interventions identified through both methods were compiled for prioritisation and categorisation into higher level themes. Clarke and Cooper's ${ }^{13}$ taxonomy was also used to guide and organise the categorisation process.

Three interviews were initially analysed independently by GW, SM and $\mathrm{BH}$. The three researchers then compared results for consensus of interpretation. All remaining interviews were analysed by GW. Once a long list of interventions and proposed higher level themes was developed by GW, a review by the full research team was conducted. At this stage, many potential interventions had been identified. Priority interventions reported in this paper were determined in the same way key themes are identified in the Braun and Clarke method. According to this method, 'keyness' of a theme is determined not strictly by size, for example, the amount of space within a particular interview or across the whole dataset, but rather whether it captures something important with respect to the research question and researcher judgement is necessary. When determining priority interventions in the present study, a number of factors were considered: the importance of the potential intervention as perceived by the interviewee, the presence of the theme in two or more cases across the dataset and the potential impact of the intervention as perceived by the researcher. Results of the analysis include priority interventions, higher level themes and selected details of interviewees' experiences, which provide rich context and insight into the value of the proposed interventions. As the focus of the study is on 


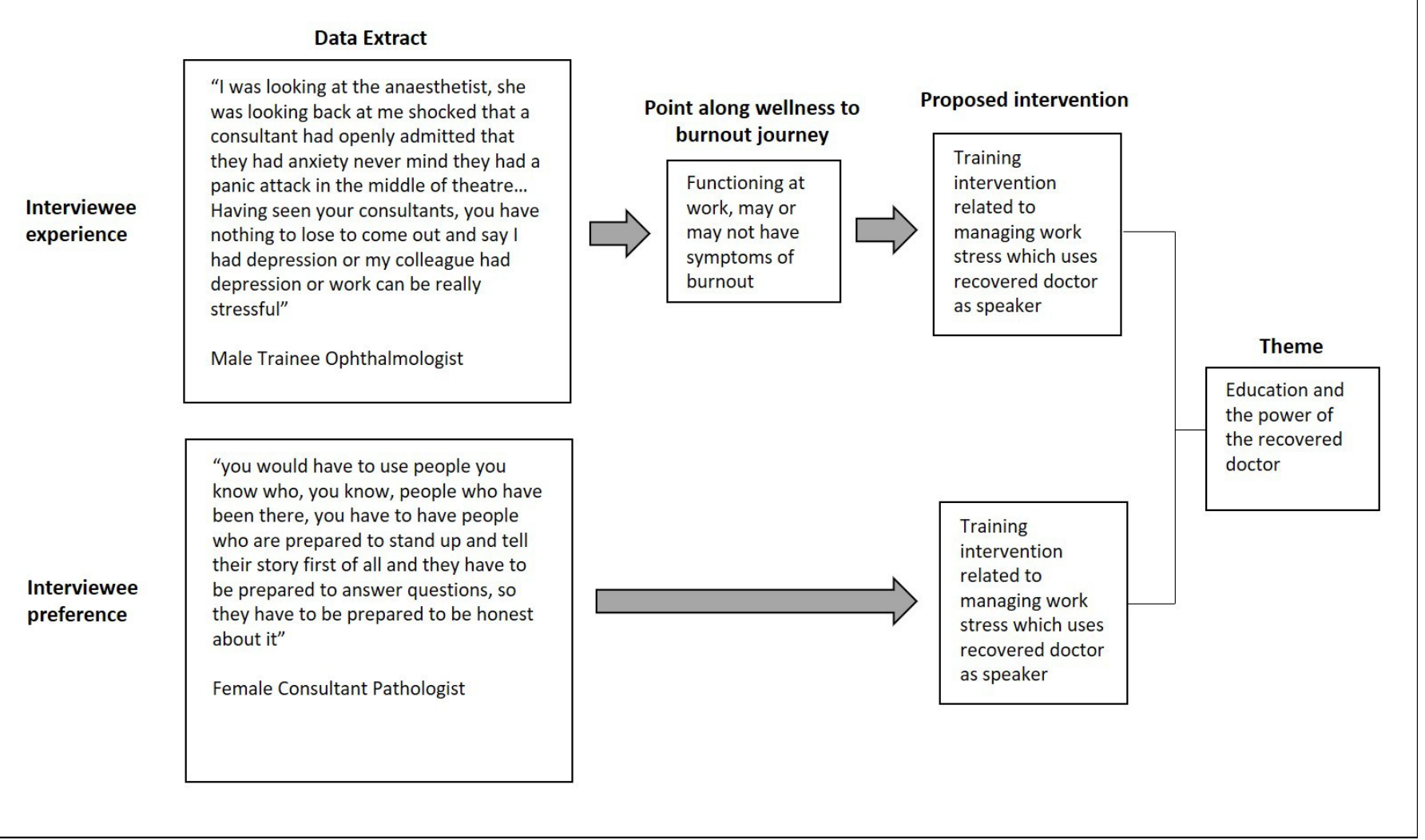

Figure 1 Example of analysis to identify potential interventions to tackle work stress and burnout using an interviewee experience and an interviewee preference.

priority interventions, unusual cases and associated minor themes are not reported. Final findings, including representative quotations, were agreed with all researchers. A combination of manual and computer-assisted (QSR NVivo10) methods was used to manage the analysis.

\section{Patient and public involvement statement}

Hospital doctors constitute the patient population in this study. The study was governed by a steering group with representation from all of the participating professional bodies. Through this forum, the study design was reviewed at different stages during its development by five practising hospital consultants and two hospital doctors in training. The interview topic guide was piloted by three hospital doctors (one consultant and two doctors in training), and they each provided feedback on the content, structure and experience of the interview.

\section{RESULTS}

\section{Work stress-related ill health reported}

During the interviews, interviewees were asked to describe any work stress-related ill health experienced. This self-reported information (figure 2) has been included in the results reported here to provide context and to serve as background to the main findings.

\section{Main findings}

These results detail the priority interventions identified through the analysis and the key themes associated with these interventions. Within the description of the key themes, details of interviewees' collective experiences, which provide context and insight into the value of the proposed interventions, are included. A summary of results can be found in table 3. Primary-level

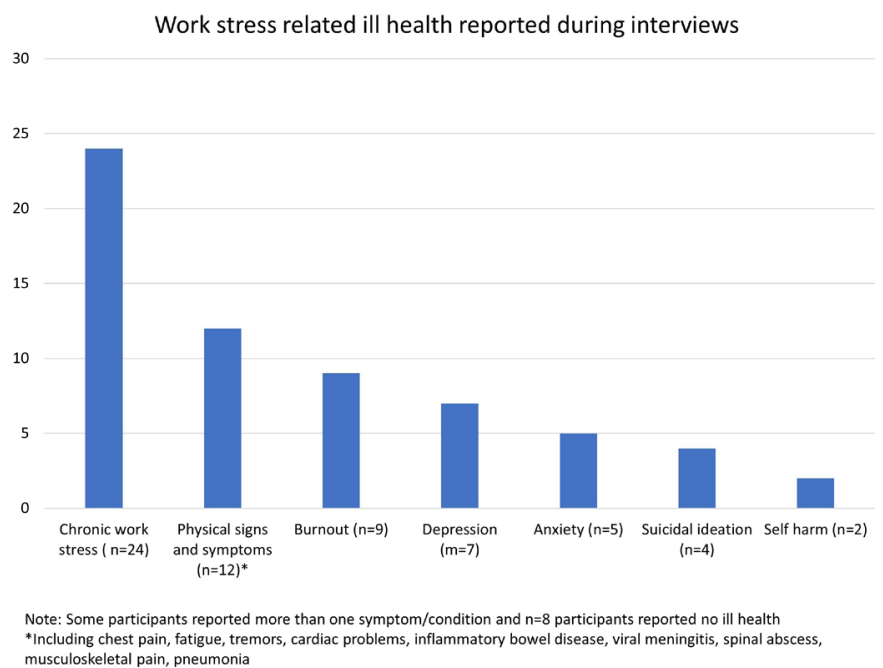

Figure 2 Work stress-related ill health reported during interviews. 
Table 3 Summary of principal findings

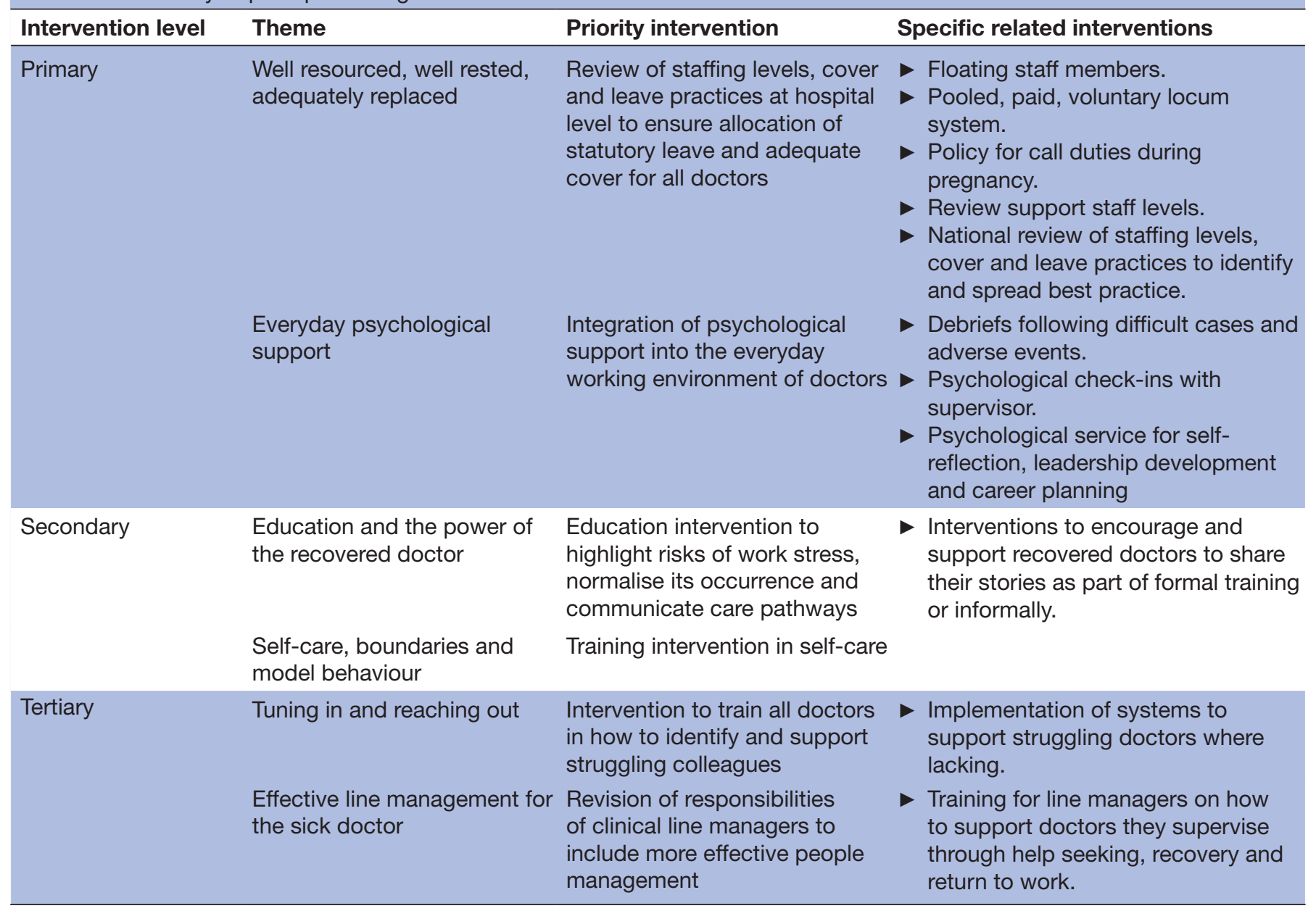

interventions to target fundamental issues contributing to increased work stress across the system were identified as higher priority than secondary-level and tertiary-level interventions.

\section{Primary-level interventions}

Well resourced, well rested and adequately replaced

Interviewees regularly described scenarios reflecting a hospital system under strain and sustained pressure to meet unmanageable demand. Staffing levels were viewed by most interviewees as unsafe for staff and patients. Human Resources (HR) practices, which force sick doctors to secure their own cover, were described and considered inadequate and unethical. Trainees, who rotate through several hospitals throughout their training, perceived workload, staffing levels and cover arrangements to be less well managed in some hospitals and highlighted this deficit as an opportunity for improvement. The intervention identified as the highest priority was a review of staffing levels and cover arrangements locally, with the goal of facilitating for all doctors the allocation of statutory leave and ensuring full cover during statutory leave.

Further interventions identified that are related to these goals include the adoption of staffing systems that allow for slack in the system, that is, floating staff members and pooled, paid locum systems, revision of procedures for applying for leave and securing cover to more clearly delineate the role of HR, implementation of a standard policy around call duties during pregnancy and an increase in non-clinical support staff numbers. A national review of staffing levels and practices around cover arrangements across hospitals was identified as a beneficial, parallel initiative.

"The system needs to build in spare capacity within existing resources to cover what are normal HR administrative practices, people go on maternity leave, people have sick leave, people have annual leave, people have CME leave". Female consultant psychiatrist

"The only way the stigma is going to be removed is if the workload of the sick individual doesn't have to be immediately transferred onto the well colleague". Male consultant geriatrician

"Knowing when your leave is going to be, being able to take your leave reasonably when you want it, that is very important". Female consultant geriatrician

"From a trainee perspective the one thing that would make a difference is if there was a policy ... you are 6 months pregnant ...you shouldn't be working 
twenty four hours in a row when about three o clock in the morning... you are ready like to pass out and it's all too serious... none of these discussions that you have to have with the rota maker...that's nonsense". Female trainee anaesthetist

\section{Everyday psychological support}

Although most interviewees highlighted the benefits of venting and sharing day-to-day problems and clinical cases with colleagues, a distinct lack of psychological support for difficult cases and adverse events, as well as more everyday challenges, was described. The analysis identified three potential interventions to meet this need. First, the introduction of regular debriefing, particularly following difficult cases, adverse events and psychological support for associated legal processes, was identified. Debriefs for everyday challenges and difficult cases should be conducted by a senior team member and should cover psychological impact, as well as clinical learning points. Doctors who supervise other doctors should be trained in debriefing. Conducting debriefs following adverse events and during associated legal processes may require a specialised skill set and an appointed person within a department who has undergone the relevant training or colleagues who have been through the process themselves would be required.

"I would work with 30 different consultants and at the end of the day maybe $10 \%$ of them would turn around and have a debrief of the day.... you go over the things that went well, the things that we can improve on... that is your take home message...if that wasn't done you would think about all the negative things". Female trainee emergency

"I know for a fact no way would any of the medical staff have any support in that regard (psychological) either, and they are just expected to just work through it and just deal with death after death without any supports". Male consultant emergency

Second, regular psychological check-ins by a supervisor or a clinical line manager as part of standard work supervision practice was identified as a key intervention to meet this need. The third intervention identified was an onsite, psychological service geared to aid self-reflection, leadership development and career planning (particularly at career transitions and life pressure points), as distinct from a treatment service.

\section{Secondary-level interventions}

Interventions designed to move hospital culture away from the potentially harmful practices of presenteeism and overcommitment towards a more compassionate culture that emphasises self-care, tolerance and support were identified as the priority interventions at a secondary level.

\section{Education and the power of the recovered doctor}

Interviewees described a culture in which performance is valued above wellness, in which doctors who struggle to cope are perceived as weak and in which doctors continue to work when physically and mentally ill. Interviewees' stories indicate that this culture, while never formally endorsed, begins in the performance-focused, competitive environment of medical school and is reinforced by senior doctors and group dynamics in clinical practice. Health promotion interventions, which educate doctors at all career stages on the likelihood and risks of work stress and burnout, normalise their occurrence and provide information on pathways to care, were identified as priority interventions. Encouraging and supporting recovered doctors to speak out publicly as part of educational programmes and informally among their peer group were identified as potentially highly effective in breaking the culture of stigma.

"This isn't something specific to a small group of people who just can't hack it. This is something that is likely to be a challenge for most doctors at some stage in their career... it's seen as a problem of the doctor rather than a kind of sensible reaction to a non-sensible situation... why don't we talk about that?" Female consultant psychiatrist

"He said he would get awful panic attacks... 'I used to get sweaty and feel like I was having a heart attack, I would have to leave the room, take off my gown and go for a walk in the cold.' He was near retirement. I said 'I had never heard of a consultant having a panic attack' and he said 'yes because no one would say it'....and I was looking at the anaesthetist, she was looking back at me shocked that a consultant had openly admitted that they had any anxiety never mind that they had a panic attack in the middle of theatre...I thought 'oh my god this is a revelation'". Male trainee ophthalmologist

\section{Self-care, boundaries and model behaviour}

Many doctors described a level of dedication to work that leaves little room for other aspects of life and may amount to overcommitment. A self-care training intervention including practical advice on prioritising and taking responsibility for your own health was identified as a priority secondary-level intervention. Key elements of a self-care training intervention should include establishing and maintaining boundaries between work and personal life, cultivating interests outside of work, and some form of spirituality or meditative practice. Such an intervention should position self-care skills as of equal importance to clinical skills and should encourage doctors to role model healthy work habits to their colleagues, particularly doctors in training. A preference for self-care interventions over resilience-building interventions was explicitly emphasised.

"There is quite a dependency, almost, on work, that work becomes the dependency. My identity and my work would be very, very closely entwined. And if you asked me my identity outside the work place, it would be less developed". Male Consultant Paediatrician 
"You need to organise your life so that you are not so stressed...endless sympathy is almost like enabling... you can't take on all this stuff, you have to say no". Female Consultant Physician

"We all bear personal responsibility and now as a consultant... I have a responsibility to the people that I work with to try and model more appropriate behaviour". Female Consultant Psychiatrist

\section{Tertiary-level interventions}

Priority tertiary interventions identified highlight opportunity and willingness among doctors to aid the recovery of sick colleagues with the help of essential procedures and training on how to support one another.

\section{Tuning in and reaching out}

Some interviewees described a lack of self-awareness in themselves and in some of their colleagues regarding the impact of work stress and burnout on their health and cited this as a reason they would not seek help. Other reasons included fear of professional consequences, feeling too busy and fear of being perceived as weak. Many described continuing to work to the point where their health problems became very advanced and serious.

Feeling tuned out, too busy or just surviving themselves, some interviewees felt they also would not notice if a colleague was having difficulties. Reflecting on times when they did notice and could have reached out to a struggling colleague, a small number of interviewees described acting confidently. Most interviewees, however, reported a lack of awareness of procedures, a lack of confidence in this area or a reluctance to help based on self-preservation. To counter this delayed help-seeking behaviour and to improve colleague-to-colleague level support, an intervention designed to train doctors, particularly those in supervisory roles, on how to identify and support struggling colleagues was identified as potentially of high value. This intervention would necessitate the development and implementation of procedures where lacking.

"I have certainly seen it (work stress and burnout) in other people you know, and at a personal level, I have tried to speak to them and I have tried to support them but there is no infrastructure". Female consultant pathologist

"You acknowledge that somebody else has a problem, then you end up de facto taking over their workload... I certainly can't do it for the number of people who I think have problems at the moment". Female consultant physician

"It should be made mandatory that the trainers should have some kind of briefing... to look out for or recognise trainees in distress". Male trainee obstetrics and gynaecology

\section{Effective line management for the sick doctor}

A need to be supported by the hospital throughout the recovery process was described, along with a need for line manager and occupational health to be aligned and for occupational health recommendations to be adhered to. Following recovery, though expressing a clear preference for a phased return to work, most participants in the study described experiences of returning to work back at full commitment or with a planned, phased reintroduction, which was not fully observed and with no follow-up. In cases where follow-up meetings were held, the emphasis was often on increasing workload rather than facilitating continued recovery.

Interviewees' stories of help seeking, recovery and return to work highlighted a management void for doctors, which leaves a considerable gap in what would be normal support structures for other employees. A review of the skill requirements of clinician line managers, which would incorporate line management skills, is needed. In relation to the specific needs of the sick doctor, a training intervention to educate supervising doctors on how to support sick doctors through the process of seeking help and rehabilitation was identified as a priority, tertiary-level intervention.

"For the first 3 months I was off, I was bringing in weekly certificates even though I was virtually bed bound by then and it took me 5 months to access my line manager, find out who it was, he hadn't been trained in what was required of him, I wasn't referred to occupational health, I had to refer myself". Female consultant

"When I was sick, there was no definitive way to get me back to the workforce in a sustainable fashion. It was all about 'get back to work, you know, you can work ten hours, you know, push, push, we will leave you on your own'". Male consultant emergency

"We both had to make up all of the calls we had missed during our 2 months off. So, then we were punished... if you can't have one of the most stressful life events and take a bit of time off if you have depression, what can you actually take time off for without being penalised?" Male trainee ophthalmologist

\section{DISCUSSION}

This study set out to identify priority interventions to prevent and reduce work stress and burnout in hospital doctors and found that practical, system-focused interventions are of greatest need. Reflecting a system under strain, challenges with basic entitlements, which most workers take for granted, such as accessing statutory leave, knowing in advance when leave can be taken and being adequately covered when on leave, were identified as requiring urgent attention. Other priority interventions identified were a broadening of the support function provided by clinical line managers to their staff, debriefs following difficult cases and adverse events, 
positive cultural change within the medical community and genuine support for self-care in the workplace, particularly at career transitions and life pressure points.

The preference of doctors in this study for primary-level, system-focused interventions over secondary-level, individual-focused training interventions is in line with preferences of doctors in Ireland uncovered in a recent study. ${ }^{34}$ It has also been shown that physicians expected to deal with their burnout individually feel less 'resilient' and take more personal ownership of their burnout than physicians who receive support from their hospital through system-level changes. ${ }^{35}$ However, to date, interventions tested and published in the literature have been predominantly individual focused. ${ }^{202}$ Which type of intervention is most effective is unclear. One recent systematic review of interventions for the prevention and reduction of burnout ${ }^{22}$ found similar improvements with both individual-level and system-level interventions. Another similar review ${ }^{23}$ found greater benefits following system-level interventions. An earlier systematic review of interventions to prevent burnout from 2010 found that a combination of individual-level and system-level interventions had longer lasting positive effects than either individual-level or system-level interventions alone. ${ }^{37}$ All reviews called for more system-level interventions to be tested. ${ }^{2022} 23$ Our study supports the predominant recommendation in the literature to focus on system-level interventions and has identified key opportunities for intervention at primary (system), secondary (individual) and tertiary (both) levels, which can be implemented in a combined approach.

The overwhelming emphasis on issues of staffing, cover and leave in our study is in line with other studies and not surprising, given the current doctor shortage and growing patient population in Ireland. ${ }^{34} 38$ The shortage of doctors is a global phenomenon predicted to reach crisis levels by $2030 .{ }^{39-42}$ Burnout has been associated with increased physician attrition, ${ }^{43}$ and as more burnt-out physicians reduce their hours, migrate and seek early retirement, those who stay are left under greater strain. However, while staff shortages are a problem for all hospitals, our study identified better staffing and leave practices in some hospitals over others, resulting in less stress for doctors in training who rotate through those hospitals. This suggests that the current situation in some hospitals could be improved through optimisation of existing management and HR practices. This finding adds further nuance to a related finding in a 2016 study of key stressors for hospital doctors in Ireland where the top three stressors for consultants were related to a lack of long-term planning, quick fix management decisions with respect to resourcing among other things and staff shortages. ${ }^{39}$

Along with adequate staffing, good leadership and support from line manager have been found to reduce the risk of burnout. ${ }^{44-46}$ The identification of lack of adequate support from line managers as a key opportunity for intervention in our study is telling the same story.
Doctors in supervisory roles are not adequately trained and do not have enough time to fulfil the people management functions of a line management role. ${ }^{47}$ Doctors all over the world are trained with an extreme emphasis on technical skills to treat patients with little time apportioned to learning the skills needed to be effective leaders or supportive managers. ${ }^{48}$ Yet this support can make a vital difference to a doctor experiencing burnout. A Dutch study into help-seeking behaviours of human service professionals identified the key, gate-keeping role of supervisors in the process of help seeking and recovery. ${ }^{49}$ This ties in with our findings, which highlight the need for doctors in the supervision of others to be able to identify struggling colleagues and to support them through help seeking, recovery and follow-up. Inadequate staffing and gaps in line management for doctors are both organisational factors that should be tackled through targeted interventions.

In addition to inadequate support from line management, cultural characteristics of the medical profession, such as an emphasis on performance, competitiveness, perfectionism and presenteeism, have been cited as triggers for mental ill health in doctors. ${ }^{50} \mathrm{~A}$ number of priority interventions identified in this study relate one way or another to the culture of medicine. A need and a willingness to move away from a culture of stigmatisation to one of support, from a culture of presenteeism and endless resilience to one of self-care and from propagation of the hidden curriculum ${ }^{51}{ }^{52}$ to the role modelling of healthy work habits were identified. While it has been argued that this kind of cultural change can only be achieved by a gradual, bottom-up approach that does not threaten existing, deeply embedded professional identities ${ }^{53}$ our study identified training interventions throughout the medical career as potentially valuable, particularly if recovered doctors are involved as facilitators. Finally, training is not the only way to bring about this cultural change. Physicians at an individual level can help by taking personal responsibility for their own health. Higher levels of reported self-care have been shown to be linked to lower levels of emotional exhaustion in doctors, ${ }^{54}$ and the related concept of self-efficacy, defined as the belief we have in our own abilities, ${ }^{55}$ has also been shown to be negatively related to burnout. ${ }^{56}$ Many aspects of medical education are now believed to occur through role modelling. ${ }^{515758}$ Senior doctors can further drive the necessary cultural change by being aware of the impact of their behaviour on junior colleagues and using this influence to model healthy and supportive work habits. The contributions on this subject of senior doctors interviewed in our study suggest that this, though challenging, is not impossible.

The main strength of this study is that it puts the physician's voice at the centre of the process of selecting and developing interventions to reduce physician work stress and burnout. The study is further strengthened by the purposive sample of interviewees selected to capture experiences and preferences of individuals from a range 
of gender, specialty and career stage, hospital type and geographical location. A limitation is that the original pool of volunteers, from which the interviewees were purposively selected, may have been a self-selected group who put themselves forward for interview because of negative personal experiences with work stress and burnout. However, arguably, these types of experiences provide most insight into opportunities for intervention. It is also worth noting that $25 \%(n=8)$ of the interviewees did not have a previous negative experience with work stress or burnout but came forward due to a general interest in the topic, which is also interesting and may signify a positive shift in attitude. Another potential limitation is that this study did not include non-consultant hospital doctors outside of training programmes and doctors in general practice. Both groups are at risk of work stress and burnout and should be included in future research.

Credibility of the study is also strengthened by the different perspectives represented by the research group ${ }^{59}$ which consists of an occupational health physician and three experienced researchers (all women) with diverse relevant expertise from the fields of human resources, organisational psychology, health psychology, human performance, health services and medical education. The interviewer's (GW) specific experience is in health services, health psychology and medical education research.

\section{CONCLUSIONS AND KEY RECOMMENDATIONS}

Hospital doctors are feeling the effects of greater demand and fewer resources. What they most urgently need is adequate staffing levels, access to statutory leave and adequate cover when on leave. It is a recommendation of this study that healthcare organisations not only intensify their focus on recruitment and retention but also review and optimise current practices around cover and leave as the highest priority. Doctors do not receive the support they need from their clinical line managers who, due to intensive clinical skills training, may lack the skills and time to excel as people managers. To rectify this, healthcare organisations, medical schools and postgraduate training bodies need to focus on providing doctors who supervise others with the time and training to perform key management activities, such as debriefs and identifying and supporting sick team members. The culture of medicine needs to change from stigmatisation and competitiveness to one of compassion and collaboration. It is incumbent on healthcare organisations, medical schools and professional bodies to steer this change through education and to engage recovered doctors, willing to share their powerful stories, as facilitators. Individual doctors can help further by acknowledging the influence they have on their junior colleagues and peers and using this influence to model compassion, self-care and healthy work habits. Researchers can contribute by comparing efficacy of interventions more rigorously and placing an emphasis on testing primary, system-level interventions and combined intervention approaches.

Acknowledgements The authors express gratitude to all the doctors who took part in these interviews and shared with us their insight and experience. We also thank our study steering group for their input into the design of this study and the doctors who participated in the pilot phase of the study.

Contributors All authors were involved in the design of the study, the design of the topic guide and the review of pilot interviews. GW conducted all the interviews and the bulk of the analysis. SM and BH conducted three interviews. All authors were involved in finalising analysis. GW wrote the first draft of the manuscript. SM, YF and $\mathrm{BH}$ contributed to subsequent drafts.

Funding We wish to acknowledge the generous financial support provided by the Human Resources National Directorate of the Health Service Executive, as well as that provided by the Royal College of Physicians of Ireland, the Royal College of Surgeons in Ireland and the College of Anaesthetists.

Competing interests None declared.

Patient consent for publication Not required.

Ethics approval Ethical approval was obtained from the ethics committee of the Royal College of Physicians of Ireland (RCPI) (RCPI RECSAF 20). A $€ 50$ gift voucher was offered to all participants as an acknowledgement of their time.

Provenance and peer review Not commissioned; externally peer reviewed.

Data availability statement Data are available upon reasonable request.

Open access This is an open access article distributed in accordance with the Creative Commons Attribution Non Commercial (CC BY-NC 4.0) license, which permits others to distribute, remix, adapt, build upon this work non-commercially, and license their derivative works on different terms, provided the original work is properly cited, appropriate credit is given, any changes made indicated, and the use is non-commercial. See: http://creativecommons.org/licenses/by-nc/4.0/.

\section{REFERENCES}

1. Shanafelt TD, Hasan O, Dyrbye LN, et al. Changes in burnout and satisfaction with work-life balance in physicians and the general us working population between 2011 and 2014. Mayo Clin Proc 2015;90:1600-13.

2. Peckham C. Physician Burnout: It just keeps getting worse. Medscape, 2015. Available: https://www.medscape.com/viewarticle/ 838437

3. Shanafelt TD, Balch CM, Bechamps G, et al. Burnout and medical errors among American surgeons. Ann Surg 2010;251:995-1000.

4. Fahrenkopf AM, Sectish TC, Barger LK, et al. Rates of medication errors among depressed and burnt out residents: prospective cohort study. BMJ 2008;336:488-91.

5. Shanafelt TD, Balch CM, Dyrbye L, et al. Special report: suicidal ideation among American surgeons. Arch Surg 2011;146:54-62.

6. Andrew BL, Brenner EB. Physician suicide. Medscape, 2018. Available: https://emedicine.medscape.com/article/806779-overview

7. Thomas S, Burke S, Barry S. The Irish health-care system and austerity: sharing the pain. Lancet 2014;383:1545-6.

8. World Health Organisation. Global health and aging, 2016. Available: https://www.who.int/ageing/publications/global health.pdf

9. Humphries N, Crowe S, Brugha R. Failing to retain a new generation of doctors: qualitative insights from a high-income country. BMC Health Serv Res 2018;18:144.

10. Humphries N, McAleese S, Matthews A, et al. 'Emigration is a matter of self-preservation. The working conditions ... are killing us slowly': qualitative insights into health professional emigration from Ireland. Hum Resour Health 2015;13:35.

11. Lazarus RS, Folkman S. Stress, appraisal and coping. New York: NY: Springer Publishing, 1984

12. Maslach C, Schaufeli WB, Leiter MP. Job burnout. Annu Rev Psycho 2001:52:397-422.

13. Clarke S, Cooper CL. Managing the risk of workplace stress. London and New York: Routelage, 2004.

14. Cooper CL, Cartwright S. An intervention strategy for workplace stress. J Psychosom Res 1997;43:7-16.

15. Sutherland VJ, Cooper CL. Strategic stress management: an organizational approach. New York: Pargrave, 2000.

16. Giga SI, Noblet AJ, Faragher B, et al. The UK perspective: a review of research on organisational stress management interventions. Aust Psychol 2003;38:158-64. 
17. Health and Safety Executive [HSE]. Tackling Work-related Stress: $A$ Managers' Guide to Improving and Maintaining Employee Health and Well-Being. Sudbury: HSE Books, 2001.

18. Health and Safety Authority. Work related stress: a guide for employers, 2009. Available: https://www.hsa.ie/eng/Publications and_Forms/Publications/Occupational_Health/Work_Related_Stress. pdf

19. Lamontagne AD, Keegel T, Louie AM, et al. A systematic review of the job-stress intervention evaluation literature, 1990-2005. Int J Occup Environ Health 2007;13:268-80.

20. Babyar JC. They did not start the fire: reviewing and resolving the issue of physician stress and burnout. J Health Organ Manag 2017:31:410-7.

21. Ahola K, Toppinen-Tanner S, Seppänen J. Interventions to alleviate burnout symptoms and to support return to work among employees with burnout: systematic review and meta-analysis. Burn Res 2017:4:1-11.

22. West CP, Dyrbye LN, Erwin PJ, et al. Interventions to prevent and reduce physician burnout: a systematic review and meta-analysis. Lancet 2016;388:2272-81.

23. Panagioti M, Panagopoulou E, Bower P, et al. Controlled interventions to reduce burnout in physicians: a systematic review and meta-analysis. JAMA Intern Med 2017;177:195-205.

24. Clough BA, March S, Chan RJ, et al. Psychosocial interventions for managing occupational stress and burnout among medical doctors: a systematic review. Syst Rev 2017;6:144.

25. Abildgaard JS, Saksvik Per $\varnothing$, Nielsen K. How to measure the intervention process? an assessment of qualitative and quantitative approaches to data collection in the process evaluation of organizational interventions. Front Psychol 2016;7:1380.

26. Lutz G, Scheffer C, Edelhaeuser F, et al. A reflective practice intervention for professional development, reduced stress and improved patient care-A qualitative developmental evaluation. Patient Educ Couns 2013:92:337-45.

27. Nissim R, Malfitano C, Coleman M, et al. A qualitative study of a compassion, presence, and resilience training for oncology interprofessional teams. J Holist Nurs 2019;37:30-44.

28. McGowan Y, Humphries N, Burke H, et al. Through doctors' eyes: a qualitative study of hospital doctor perspectives on their working conditions. Br J Health Psychol 2013;18:874-91.

29. Turk M, Davas A, Tanik FA, et al. Organizational stressors, workfamily interface and the role of gender in the hospital: experiences from Turkey. Br J Health Psychol 2014;19:442-58.

30. Liu C-H, Tang W-R, Weng W-H, et al. The process of coping with stress by Taiwanese medical interns: a qualitative study. BMC Med Educ 2016;16:10,016-534.

31. Clarke V, Braun V. Successful qualitative research: a practical guide for beginners. London: SAGE, 2013.

32. Hayes B, Prihodova L, Walsh G, et al. What's up doc? A national cross-sectional study of psychological wellbeing of hospital doctors in Ireland. BMJ Open 2017;7:e018023.

33. Braun V, Clarke V. Using thematic analysis in psychology. Qual Res Psychol 2006;3:77-101.

34. Feeney S, OBrien K, OKeeffe N, et al. Practise what you preach: health behaviours and stress among non-consultant Hospital doctors. Clin Med 2016;16:12-18.

35. Ruotsalainen $\mathrm{JH}$, Verbeek JH, Mariné A, et al. Preventing occupational stress in healthcare workers. Cochrane Database Syst Rev 2015;184.

36. West CP, Dyrbye LN, Rabatin JT, et al. Intervention to promote physician well-being, job satisfaction, and professionalism: a randomized clinical trial. JAMA Intern Med 2014;174:527-33.

37. Awa WL, Plaumann M, Walter U. Burnout prevention: a review of intervention programs. Patient Educ Couns 2010;78:184-90.
38. Hayes B, Fitzgerald D, Doherty S, et al. Quality care, public perception and quick-fix service management: a Delphi study on stressors of hospital doctors in Ireland. BMJ Open 2015;5:e009564.

39. Williams D, Thomas S. The impact of austerity on the health workforce and the achievement of human resources for health policies in Ireland (2008-2014). Hum Resour Health 2017; 15:62,017-230.

40. OECD. International Migration of Doctors. In: Health at a glance. OECD iLibrary, 2015

41. Association of American Medical Colleges. The complexities of physician supply and demand: projections from 2016 to 2030 (2018 update), 2018. Available: https://aamcblack.global.ssl.fastly.net/ production/media/filer_public/85/d7/85d7b689-f417-4ef0-97fbecc129836829/aamc_2018_workforce_projections_update_april_11_ 2018.pdf

42. Liu JX, Goryakin Y, Maeda A, et al. Global health workforce labor market projections for 2030. Hum Resour Health 2017;15:11,017-187.

43. Hamidi MS, Bohman B, Sandborg C, et al. Estimating institutional physician turnover attributable to self-reported burnout and associated financial burden: a case study. BMC Health Serv Res 2018;18:851.

44. Gibson JA, Grey IM, Hastings RP. Supervisor support as a predictor of burnout and therapeutic self-efficacy in therapists working in ABA schools. J Autism Dev Disord 2009;39:1024-30.

45. Hämmig O. Health and well-being at work: the key role of supervisor support. SSM Popul Health 2017;3:393-402.

46. Willemse BM, de Jonge J, Smit D, et al. The moderating role of decision authority and coworker- and supervisor support on the impact of job demands in nursing homes: a cross-sectional study. Int J Nurs Stud 2012;49:822-33.

47. Ireri S, Walshe K, Benson L, et al. A qualitative and quantitative study of medical leadership and management: experiences, competencies, and development needs of doctor managers in the United Kingdom. J Manag Marketing Healthc 2011;4:16-29.

48. Montgomery A. The inevitability of physician burnout: implications for interventions. Burn Res 2014;1:50-6.

49. Putnik K, de Jong A, Verdonk P. Road to help-seeking among (dedicated) human service professionals with burnout. Patient Educ Couns 2011;83:49-54.

50. Devi S, Distress Din. Doctors in distress. Lancet 2011:377:454-5.

51. Hafferty FW. Beyond curriculum reform: confronting medicine's hidden curriculum. Acad Med 1998;73:403-7.

52. Hafler JP, Ownby AR, Thompson BM, et al. Decoding the learning environment of medical education: a hidden curriculum perspective for faculty development. Acad Med 2011;86:440-4.

53. Mintzberg H. Toward healthier hospitals. Health Care Manage Rev 1997;22:9-18.

54. Zhu W, Wang ZM, Wang MZ, et al. Occupational stress and job burnout in doctors. Journal of Sichuan University. Medical science edition 2006;37:281-3.

55. Bandura A. Self-Efficacy: the exercise of control. New York: H Freeman, 1997

56. Schwarzer R, Hallum S. Perceived teacher self-efficacy as a predictor of job stress and burnout: mediation analyses. Appl Psychol 2008;57:152-71.

57. Wright SM, Carrese JA. Excellence in role modeling: insights and perspectives from the pros. Can Med Assoc J 2002;167:638-43.

58. Wright S, Wong A, Newill C. The impact of role models on medical students. J Gen Intern Med 1997:12:53-6.

59. Graneheim UH, Lundman B. Qualitative content analysis in nursing research: concepts, procedures and measures to achieve trustworthiness. Nurse Educ Today 2004;24:105-12. 